\title{
Light waves, radio waves and photons
}

\author{
BY \\ RICHARD M. SILLITTO \\ B.Sc., F.Inst.P. \\ (University of Edinburgh)
}




\title{
Light waves, radio waves and photons RICHARD M. SILLITTO
}

\author{
B.Sc., F.Inst.P. \\ University of Edinburgh
}

[A lecture given to the Scottish Branch in Edinburgh on November 9, 1959, and in Glasgow on November 10]

$\int^{N}$ N 1900 Planck solved the problem of constructing a formula to represent the distribution in energy of the radiation in a uniform temperature enclosure. The formula he obtained seemed to imply that the exchange of energy between the radiation field and the walls of the enclosure must take place in discrete amounts or quanta, the size of the quantum at the (angular) frequency $\omega$ being $\hbar \omega$, where $\hbar \approx \mathbf{1 0}^{-27}$ erg sec. Einstein's subsequent interpretation of the photoelectric effect (1905) also rested on the assumption that light of (angular) frequency $\omega$ is absorbed by a photosensitive surface in amounts $\hbar \omega$. Whether light energy actually travels in the form of bundles or quanta was open to question, but there was no reason to suppose that it does not.

In this lecture I want to talk about some of the experiments which were expected --or might have been expected- to demonstrate quantum structure in the electromagnetic field.

Two such experiments were reported in the Proceedings of the Cambridge Philosophical Society in 1909, one by G. I. Taylor, the other by N. R. Campbell, and some very recent experiments of considerable refinement have close affinities in principle to one or other of those experiments of 50 years ago.

Taylor's experiment(1) was intended to test a suggestion made in 1907 by J. J. Thomson(2) : that the phenomena of ionization by X-rays -- and also, we may add, of photoelectricity- might be understood if the energy of electromagnetic waves was supposed to be concentrated in narrow tubes, rather than spread uniformly over a wavefront. The intensity of the radiation would be represented by the average density of the tubes, while even in a radiation field of low intensity the concentration of energy in one such tube could be high enough for an atom to pick up the energy required for ionization very quickly. Now according to the wave theory of light the diffraction pattern formed on a screen by light passing an opaque object can be calculated by summing the effect due to those zones of the wavefront which pass very near the obstacle. In making this calculation it is assumed that the amplitude of the wave is uniform over these zones. Thomson had the idea that if the light intensity was reduced sufficiently the number of tubes passing through each zone would be so small that there would be marked fluctuations of intensity from zone to zone and consequently the appearance of the diffraction pattern would be altered - the visibility of the pattern would presumably be reduced. G. I. Taylor therefore photographed the diffraction pattern of a needle for a range of light intensities: at the lowest intensity he used, an exposure of three months was needed. "In no case," he reported, "was there any diminution in the sharpness of the pattern." During his longest exposure the mean energy density of radiation within the apparatus was about $1.6 \times 10^{-16} \mathrm{ergs} / \mathrm{c} . \mathrm{c}$. (or about $2 \times 10^{-4}$ of a Planck quantum). "According to Sir J. J. Thomson," Taylor's paper concluded, "this value sets an upper limit to the amount of energy contained in one of the indivisible units [i.e. tubes of radiation] mentioned above."

This experiment is of great importance for the interpretation of quantum theory, and after the advent of quantum mechanics in 1925 two experiments of a more critical design were performed by Dempster and Batho (1927). The first of these showed that the diffraction images formed by an echelon grating retained their sharpness when the light intensity was reduced so that on average less than one quantum at a time was passing through the diffraction apparatus. Since the entrance aperture of each plate of the echelon grating was about $32 \mathrm{~mm}^{2}$ this set a lower limit to the area of the wavefront which a quantum appears to occupy; in passing we may note that this minimum area is enormously greater than the area of the atom to which the quantum can give up its energy in the photoelectric process. Dempster and Batho's second experiment showed that a normal interference pattern was formed by the partial reflection of light from two slightly inclined glass plates with a light source so weakly excited that only one atom at a time was radiating in any direction.

Most recently this question of the interference of light at very low intensities has been reinvestigated in Budapest by Jánossy and Náray(4)(1957). They built, in a tunnel 30 metres underground, a Michelson inter- 
ferometer whose arms were almost 14 metres long, enormously greater than any characteristic length such as a coherence length that might be assigned to a quantum. If interference effects appear with such an apparatus for light intensities so low that only one photon at a time can be passing through the apparatus, the formation of the interference pattern can be interpreted neither in terms of interference between different photons, nor in terms of a pseudo-classical model of photons as wavepackets of limited extent. The interference fringes were recorded by scanning a photomultiplier tube across the interference pattern, and were found to be independent of light intensity. The work of Jánossy and Náray is an outstanding piece of experimentation and verifies conclusively the result first obtained by G. I.. Taylor.

The results of this sequence of experiments might be summarized by saying that optical interference is a "single quantum process," in the sense that photons do not interfere with one another to produce interference patterns. It is interesting that an analogous conclusion can be stated for electron interference and diffraction. Our President, Sir George Thomson, pointed out in his book The wave mechanics of free electrons (5), and it was verified in 1949 by Fabrikant et al (6), that sharp electron diffraction patterns are obtained with electron beams so weak that only one electron at a time passes through the apparatus.

Now let us return again to the year 1909, and consider the experiment designed by N. R. Campbell to investigate "discontinuities in light emission." The two papers(7) in which he reported this experiment provide fascinating reading, and not only because the first
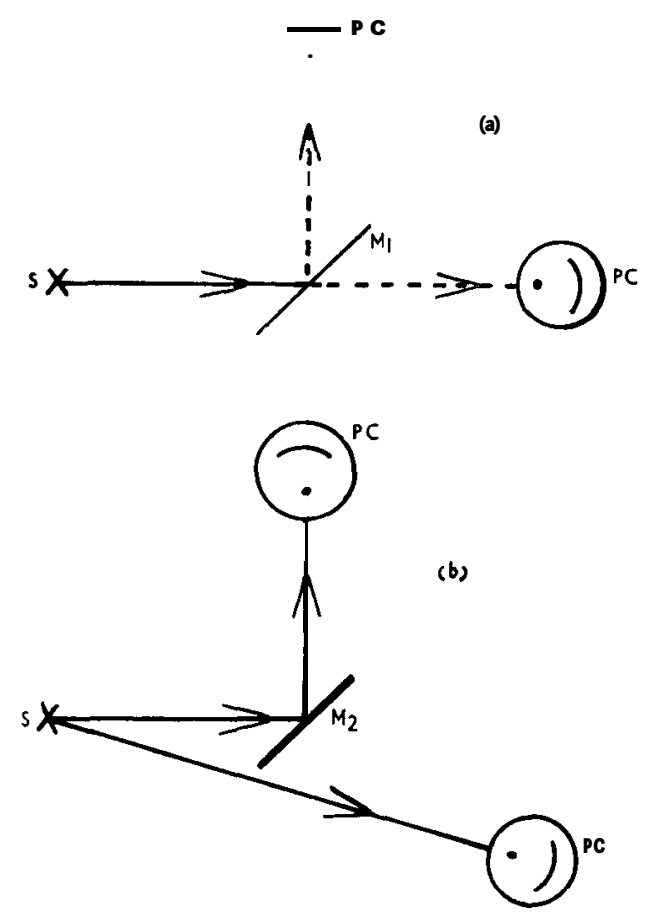

Fig. 1. S--light source ; PC photoclectric cell ; MI-halfsilvered mirror ; M 2-mirror. begins: "This is an account of an experiment which failed," and the second reported that "the experiment has continued unsuccessful"! After explaining that such a report has value, if only as a warning to others, Campbell analyzed with beautiful clarity the implications for what we would call "coherence" of Thomson's idea of tubes of radiation, and of Stark's bundle-of-energy interpretation(8) of the quantum hypothesis. $\mathrm{He}$ then suggested that the coherence of two beams of light might be investigated by a study of the correlation between the fluctuations in the outputs of two photoelectric cells placed one in each beam -- a proposal which, in those very early days of the study of discontinuous phenomena, could surely have originated with no one but N. R. Campbell. Referring to Figure 1, it seemed that if Thomson's theory were correct the beams falling on the photocells in the arrangement of Figure la might be "dependent", while in the arrangement of Figure $1 \mathrm{~b}$ they might be "independent"; on the bundle-of-energy theory -- which resembled the rather crude photon model which still we sometimes use too naively -- Campbell thought the correlations in the two arrangements would be the same. With the two cells connected as in Figure 2, and the potentiometer balanced, any light fluctuation

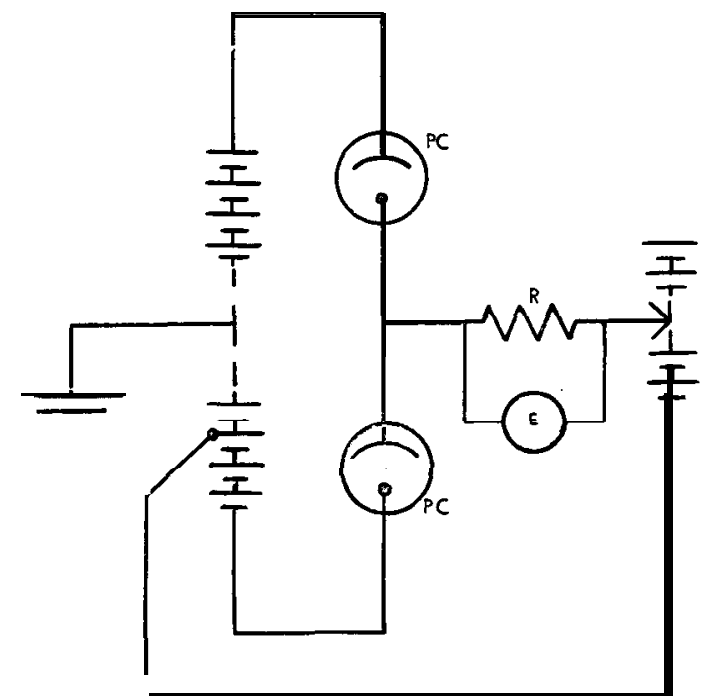

Fig. 2. PC-photoelectric cell; R- high resistance; E- electrometerr.

which affected both cells simultaneously would not affect the balance, and no current should flow in the high resistance $R$, but uncorrelated fluctuations in the two beams would cause a fluctuating voltage to develop across $R$ and so would affect the electrometer. Campbell's experiment failed -- as he shows in papers which analyse carefully and frankly its every aspect, theoretical and technical -- because of the erratic behaviour of his light sources, and all his efforts could not overcome this technical difficulty. But what a brilliantly conceived experiment, and what a remarkable pair of papers!

Within the last ten years, the effects which Campbell hoped to investigate have been studied intensively, and the interpretation of the experimental results was for a 
year or two the subject of controversy. If the photocells of Fig. la are replaced by photomultiplier tubes, evidence for the possible correlation between the outputs of the photomultipliers may be sought in two ways. In the first, suggested by Bay and realized by Adám, Jánossy and Varga(9), the pulses produced at the anodes by the emission of individual photoelectrons from the cathodes are fed to counters, and also to a coincidence detector. If the resolving time of the coincidence detector is known it is easy to calculate, from the counting rates in the two photomultipliers, the coincidence rate which should be observed if events in the two counters are random and uncorrelated. Any excess of the observed coincidence rate over the calculated coincidence rate will then show that there is a positive correlation between the outputs of the two multipliers. In the second detection technique, which uses higher light intensities, the alternating components of the photomultiplier output currents are fed into a correlation motor -- in effect a sensitive integrating a.c. wattmeter whose two windings are fed from the outputs of the two photomultipliers -- and any correlation between the photomultiplier outputs will cause the fluctuations in position of the motor shaft to be about a mean which moves progressively in one direction. If the correlation is small its effect may not be seen : in the first arrangement because it may be masked by the random fluctuations in the counting rates, in the second arrangement because the random fluctuations of the position of the motor shaft may conceal the slight advance of its mean position. In fact the first experiment by Jánossy and his collaborators gave a null result for this reason, and it was Brown and Twiss(10), using the second technique, who demonstrated that there is a positive correlation between the photomultiplier outputs, provided the beams falling on the cathodes are coherent, i.e. capable of producing an interference pattern if superposed; further the magnitude of the correlation depends on the degree of coherence of the light beams in the same way as does the visibility of the interference pattern they would produce if recombined. Subsequently the coincidence-detector technique was improved by a number of workers(11), and gave results consistent with those first obtained by Brown and Twiss.

It is one of the interesting features of this result that it cannot be understood in terms of the crude -- too crude! -- model of a beam of light as a stream of discrete, indivisible, corpuscular photons, just as the result of "Taylor's experiment" cannot be understood in terms of this model ; but like Taylor's observation of interference at very low light intensities the observation of Brown and Twiss can be explained in a quite classical way. Indeed we can say that it is in the nature of the quantum theory of radiation that the classical explanation is the correct one. When a beam of electromagnetic waves is split by a half-silvered mirror any intensity fluctuations in the beam, arising from the light source, propagate in both components of the split beam. They should therefore be expected to give rise to correlated fluctuations in the photocurrents generated when the two beams fall on photosensitive cathodes. The quantal nature of the photoelectric interaction shows in the fact that the photocurrent does not vary in exact proportion to the instantaneous light intensity, but depends on the intensity in a way which is only statistically determinate. What is proportional to the intensity is not the rate of emission of photoelectrons, but the probability of emission of photoelectrons. Consequently the photocurrent tends to follow the fluctuations in the intensity of the electromagnetic field, but there are also random (Poissonian) fluctuations in the photocurrent, which we call "shot noise." The correlated fluctuations are difficult to observe, because even under the best experimental conditions they tend to be small compared with the uncorrelated shot noise fluctuations.

The classical description of the effect of the fluctuations in the field intensity uses methods familiar in the theory of random signals(12). If the normalized spectral density of the radiation is $g(v)$, and if $\rho(\tau)$ is the Fourier Transform of $g(v)$, the temporal fluctuations of the field intensity $P$ can be characterized by the correlation function

$$
\overline{P(t) P(t+\tau)}=\bar{P}^{2}\left(1+|\rho|^{2}\right) .
$$

If a plane-polarized field of intensity $P$ ejects $\alpha P \delta t$ photoelectrons from a cathode in time $\delta$ t - N.B dassicalcausality is implied here! -- the mean number of photoelectrons ejected in time intervals of duration $T$ is

$$
\bar{n}_{T}=\alpha \bar{P} T \text {, so that } \bar{n}_{T}^{2}=\alpha^{2} \overline{P^{2}} T^{2} \text {. }
$$

On the other hand

$$
\begin{aligned}
\overline{n_{T^{2}}} & =\alpha^{2} \int_{0}^{T} \int_{0}^{T} P(t) P\left(t^{\prime}\right) d t d t^{\prime} \\
& =a^{2} \iint P(t) P(t+\tau) d t d \tau
\end{aligned}
$$

(within the corresponding limits)

$$
=\alpha^{2} \overline{P^{2}} T^{2}+\alpha^{2} \overline{P^{2}} T \int_{0}^{T}|\rho|^{2}\left(1-\frac{\tau}{T}\right) d \tau
$$

Now if ${ }^{T}|\rho|^{2} d \tau$ is written as $\tau_{0}$, and if $\tau_{0} \ll T$ so that the second term under the integral may be neglected, this becomes

$$
\overline{n_{T}^{2}}-\bar{n}_{T}^{2}=\alpha^{2} \bar{P}^{2} T^{2} \times \frac{\tau_{0}}{T}=\bar{n}_{T}^{2} \tau_{0} / T,
$$

which is the mean square fluctuation in the electron emission due to the fluctuations in the field, which arise in practice from the random nature of the light emissions. If then we add to this "classical" fluctuation a "quantal" term to account for the random character of the photoelectric effect, which is a Poissonian process with variance $\bar{n}_{T}$, we get finally

$$
\overline{\sigma_{T}^{2}}=\bar{n}_{T}+\bar{n}_{T}^{2} \tau_{0} / T=\bar{n}_{T}\left(1+\bar{n}_{T} \tau_{0} / T\right) \text {. }
$$

It is worth remarking on the similarity of this result to the quantum-statistical expression(13) for the mean square fluctuation in a radiation field of frequency $v$ :

$$
\overline{\frac{\Delta E^{2}}{h^{2} v^{2}}}=\mathcal{N}_{p}+\mathcal{N}_{p}^{2} / \mathcal{N}_{v} \text {. }
$$

Here $\mathcal{N}_{p}=\bar{E} / h \boldsymbol{v}$ is the number of quanta present, so $\overline{\Delta E^{2}} / h^{2} v^{2}$ can be written as $\overline{\Delta \mathcal{N}_{p}^{2}}$, and we have

$$
\overline{\Delta \mathcal{N}_{p}^{2}}=\mathcal{N}_{p}+\mathcal{N}_{p}^{2} / \mathcal{N}_{v}=\mathcal{N}_{p}\left(1+\mathcal{N}_{p} / \mathcal{N}_{v}\right) \text {. }
$$


$\mathcal{N}_{v}$ is the number of wavemodes of frequency $v$ which are available in the space occupied by the field, so the ratio $\mathcal{N}_{p} / \mathcal{N}_{v}$ indicates the degree of degeneracy of the radiation. On the other hand the ratio $\tau_{0} / T$ of equation (1) involves the "coherence time" $\boldsymbol{\tau}_{\boldsymbol{0}}$, so equations (1) and (2) together show a connection between optical coherence and the quantal property of degeneracy.

Equation (1) was first obtained by Purcell(14); for unpolarized light it becomes

$$
\overline{\mathrm{An}^{2}}=\bar{n}\left(1+\frac{1}{2} \tilde{n} \tau_{0} / T\right)
$$

since the fluctuations in orthogonal polarizations are independent. From this Purcell showed that in coincidence-counting experiments the additional waveinteraction term in (1)' -- additional, that is, to the Poissonian fluctuation represented by the first term would increase the coincidence rate by a factor $\left(1+\tau_{0} / 2 T\right)$, which would be small since in practice the coherence time for intense light sources is very small compared with the resolving time $\mathcal{T}$ of the detector circuits. In the correlation experiment of Brown and Twiss the wave-interaction fluctuation gives a crosscorrelation $\overline{(\Delta n)_{1}(\Delta n)_{2}}=\frac{1}{2} \bar{n}^{2} \quad \tau_{0} / T$ between the outputs of the two multipliers; again this term is small.

It must be emphasized that these effects arise from the classical wave features of the radiation field, and they are derived and discussed by Jánossy in a paper entitled "On the classical fluctuations of a beam of light" (15); the classical calculation assumes that the Fourier components of the electromagnetic field can be freely superposed, and this supposition is consistent with quantum mechanics since radiation quanta obey Bose-Einstein statistics.

Another class of experiments which illustrate optical correlation is concerned with the production of beats between light waves of slightly differing frequencies. In 1955 Forrester, Gudmundsen and Johnson reported an experiment(16) showing beats between the components into which the $\mathrm{Hg}$ green line $\left(A=5461 \mathrm{~A}^{\circ}\right)$ is split in a magnetic field. The beat frequency was in the microwave region, and was detected by feeding the output from a photocell into a microwave receiver. The coherence of the radiation was very low : the signal:noise ratio was only $10^{-4}$, and the observation of the effect was a remarkable achievement. In 1959 a rather different and very elegant experiment by Dodd, Fox, Series and Taylor was reported(17), which showed optical beats with a high degree of coherence; in this experiment coherence is obtained by controlling with an intense r.f. field the phases of the excited atomic states whose radiations subsequently beat together.

The results of all these experiments lead to the conclusion that interference and correlation experiments can yield no information about the quantum character of the electromagnetic field. Indeed, there are good quantum mechanical reasons for this, pointed out by Heisenberg thirty years ago in his Princeton lectures. In rather simpler terms, the argument can be stated thus:

Between the conjugate energy $(E)$ and time $(t)$ variables there is an uncertainty relation $\Delta \mathrm{E} . \Delta \mathrm{t} \geqslant \hbar$, stating a limitation, inherent according to quantum mechanics, on the degree of precision with which we can specify the state of a system characterized by these variables. If the system is a radiation field of (angular) frequency $\omega$, its energy $\mathrm{E}$ is $n \hbar \omega, n$ being the number of quanta present, while the phase $(\phi)$ of the field is related to time through $t=\phi / \omega$. Then the above uncertainty relation can be written

$$
\Delta n . \Delta \varphi \geqslant 1 \text {. }
$$

If we now consider two light beams of the same frequency a relation of this type defines the limitations on our knowledge of the state of each beam :

$$
\begin{aligned}
\Delta n_{1} \cdot \Delta \varphi_{1} & =k \text { (say) } \\
\text { and } \Delta \mathrm{n}_{2} \cdot \Delta \varphi_{2} & =k .
\end{aligned}
$$

If the two beams are superposed, the total number of quanta present is $\mathcal{N}=n_{1}+n_{2}$, and the relative phase is $\boldsymbol{\Phi}=\varphi_{1}-\varphi_{2}$; then

$$
\begin{aligned}
\Delta \mathcal{N} \cdot \Delta \Phi & =\Delta\left(n_{1}+n_{2}\right) \ldots \Delta\left(\varphi_{1}-\varphi_{2}\right) \\
& =\Delta n_{1} \cdot \Delta \varphi_{1}-\Delta n_{2} \cdot \Delta \varphi_{2}-\Delta n_{1} \cdot \Delta \varphi_{2}+\Delta n_{2} \cdot \Delta \varphi_{1} .
\end{aligned}
$$

The last two terms vanish, for anything else would imply that the measurement of phase or energy in one beam of light affects the state of a different beam, which would be absurd. Hence

$$
\begin{aligned}
\Delta \mathcal{N} . \Delta \Phi & =\mathrm{An}_{1} . \Delta \varphi_{1}-\Delta n_{2} \cdot \Delta \varphi_{2} \\
& =k-k \\
& =0 .
\end{aligned}
$$

Thus the total energy and relative phase of two interfering light beams may be measured with the precision allowed by classical wave physics, and any experiment which measures these variables, such as an interference or correlation experiment, will give the results predicted by classical theory.

Heisenberg concluded(18) : "the classical wave theory is sufficient for the discussion of all questions of coherence and interference."

In radio waves quantal effects should be even more difficult to detect, for the quantum energy is very small -about $10^{-5} \mathrm{eV}$ at centimetre wavelengths, compared with $2 \mathrm{eV}$ in the visible spectrum -- and the density of quanta in detectable r.f. fields is rather high. The random motions of the electrons in a receiving aerial generate a noise signal, whose power in the bandwidth $\delta \omega$ is $k T \delta \omega / 4 \pi$ when the aerial temperature is $T^{\circ} K$. At room temperature this amounts to about $10^{-21} \times \delta \omega$ watts. If a signal received by the aerial is to exceed the noise-rate of energy transfer between aerial and field the number of quanta of angular frequency $\omega$ transferred to the aerial by the signal in each second must exceed

$$
\frac{k T \delta \omega}{4 \pi \hbar \omega} \approx 10^{13} \times \frac{2}{\sigma}
$$

where Q, the quality factor of the aerial, might be about 100. The response time of the aerial is $2 \pi \mathrm{Q} / \omega$, so the number of signal quanta absorbed in the response time must be greater than $10^{14} / \omega$. Even at microwave frequencies, where $\omega \approx 10^{11}$, this number of quanta is quite large. Now if the signal is to have a definable phase the energy $E$ in the signal must be large enough to 
enable time measurements to be made with a precision very much better than one period, i.e. $\Delta t=2 \pi / n \omega$ where $n$ is a large number. The uncertainty relation for energy and time then shows that

$$
\Delta E \geqslant \frac{n \hbar \omega}{2 \pi}
$$

i.e. the field must contain a large number of quanta. Since the field we are concerned with here is the field interacting with the detector, this means that a large number of quanta must fall on the aerial during its response time. As we have seen, a radio signal giving a signal:noise ratio greater than one satisfies this requirement, so a single radio beam has in practice a measurable phase, and can be described macroscopically, i.e. in the language of classical physics.

Nevertheless experiments have been made at radio frequencies in the hope of exhibiting quantal effects. Some of the experimenters have argued thus :

"Elementary quantum theory shows that bound material systems-atoms and molecules, for instancehave discrete energy levels. The emission of quanta with sharp energies from such systems does not then establish unequivocally that quantum structure is an essential property of radiation ; it may merely show that in certain circumstances information about the quantization of the material system can be propagated electromagnetically. The only measure ments in the optical region which are not vulnerable to this attack are measurements of photoemission, in which although the ejected photoelectrons are free electrons their energy is nevertheless determined by the frequency of the incident light ; and measurements of the black-body radiation spectrum, for here the shape of the spectrum is independent of the materials in the walls of the cavity and must therefore reflect an intrinsic property of radiation. Further direct evidence of the quantum structure of electromagnetic radiation should therefore be sought in the interaction between electromagnetic fields and free electrons." (The Compton effect, of course, furnishes such evidence.)

One such experiment was performed by Farago and Marx (1954)(19), who fired a beam of electrons across an excited microwave cavity and examined the spreading of the emergent beam. If the electron beam is fired through the cavity in the z-direction, and the cavity is excited in a $T E_{0 m n}$ mode in which the electric field is in the $x$-direction and is independent of $x$, the $x$-component of the force on an electron is $-\boldsymbol{e}(\mathbf{E}+\mathbf{V} \wedge \mathbf{B})$, which is easily shown to be $-\boldsymbol{e} \frac{d \mathbf{A}}{d t}, A$ being the vector potential. The net $x$-component of momentum imparted to an electron during the transit of the cavity is then

$$
p_{x}=\int_{\text {transit }} F_{x} d t=-e \int_{\text {trarsit }} d \mathbf{A}=0
$$

the integral is zero because the vector potential vanishes at the boundaries of the cavity. Hence, although the electric and magnetic forces acting on the electrons might broaden the beam, they would in the end produce no change in the direclions of the electron trajectoriesat any rate according to this classical argument.

But suppose one argues(20) that the interaction between an electron and the field takes place through the absorption and emission of quanta of energy h $\omega$ : these are quanta of radiation polarised in the $x$-direction, and must therefore modify the $x$-component of the momentum of the electron. If the elementary acts of emission and absorption are independent, the number imparting " upward " momentum might not exactly balance the number imparting " downward " momentum during the transit of the electron, and for this reason the emergent beam would show an angular divergence. In fact this is not observed. The elementary emission and absorption events are not independent, being induced transitions controlled by the macroscopic field in the cavity, and a rigorous quantum mechanical discussion by Fogarassy(21) has shown that the null result of the experiment is to be expected.

A quite different type of radio frequency experiment was proposed by L. B. Slepyan (1957)(22). His suggestion was that observations should be made of the strength of the signal received from a transmitter by a very sharply tuned receiving aerial, and particularly of the way in which the received signal strength varies as the transmitted power is reduced to a very low level. Picturing the incoming signal as a stream of photons Slepyan argued that at low intensities the power spectrum of this train of impulses would broaden, because of their random distribution in time, and that a decreasing proportion of the received power would lie within the pass-band of the aerial as the photons became more widely spaced. He calculated that the effect might appear in a $3 \mathrm{~cm}$ wavelength receiver whose pass-band was less than 24 cycles/ sec.-a bandwidth which is not unattainably $\operatorname{low}(16)(23)$.

In fact, though, Slepyan's picture of the working of a radio aerial is not in accordance with quantum mechanics, which gives a much more classical account of what happens. It was pointed out by Fürth in 1951(24) that the reception of signals by a radio aerial is an induced absorption under the action of a macroscopic field round the aerial. We can show from quantum mechanical arguments(24)(25) that the net rates of emission and absorption of energy by an assembly of electrons in, say, the walls of an aerial cavity are respectively

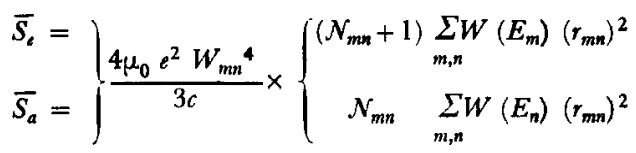

where $W(E)$ represents the distribution-in-energy of the conduction electrons in the aerial, $\boldsymbol{e r}_{\boldsymbol{m} n}$ is the dipole moment of a conduction electron for a process in which a quantum of energy $\hbar \omega_{m x}$ is emitted or absorbed, and the summation is over all the possible transitions between pairs of states whose energies differ by $\hbar \omega_{m n} ; \mathcal{N}_{m n}$ is the number of quanta of the appropriate frequency in the cavity. Suppose the size of the cavity is such that its fundamental frequency is $\omega$. When a signal of the frequency $\boldsymbol{\omega}$ is incident on the cavity the number of quanta of that frequency in the cavity grows with the time-constant of the aerial circuit, which is $2 \pi Q / \omega$. 
A dynamic equilibrium, between the number of quanta entering the cavity both from the incident radiation field and from the cavity walls, and the number removed from the cavity by absorption, is attained when the number of quanta accumulated in the cavity is very nearly equal to the number entering it in the response time $2 \pi Q / \omega$. As we have already seen this number is large if the signal:noise ratio is greater than one, and under these conditions the aerial and the field with which it interacts behave in a classical way ; in fact it is generally true that classical behaviour is observed whenever the probability of induced transitions is enormously greater than the probability of spontaneous transitions (i.e. whenever $\mathcal{N}_{m n} \gg 1$ in equation (3) ) and this will always be so in radio frequency experiments at normal temperatures.

The main conclusion from all these experiments is that the domain of validity of classical electromagnetic theory is very extensive, and that we should be very hesitant about accepting arguments which rely on the "corpuscular photon" model -- at any rate beyond the point where the photons are required to do more than obey the laws of conservation of energy and momentum. In fact, I think that if we abolished the word "photon" from our vocabulary for ten years, we should find that we could get on perfectly well without it.

\section{REFERENCES}

1. Taylor, G. I., Proc. Camb. Phil. Soc., 15 (1909), p. 114.

2. Thomson, J. J., Proc. Camb. Phil. Soc, 14 (1987), p. 417.

3. Dempster, A. J., and Batho, H. F., Phys. Rev. 86 (1927), p. 644 .

4. Jánossy, L., and $\mathbf{N}$ áray, Zs., Hungarian Academy of Sciences: Central Research Institute of Physics report (1957).
5. Thomson, G. P., The wave mechanics of free electrons (McGraw Hill. 1930). p. 119.

6. Fabrikant et al., Dokladi Akademi Nauk, 56 (1949), p. 185.

7. Campbell, N. R., Proc. Camb. Phil. Soc., 15 ( 1909), p. 310 and p. 513.

8. Stark, J., Phys. Zeits., 10 (1909), p. 579.

9. Adám, A., Jánossy, L., and V arga, P., Ann. der Physik, 16 (1954), p. 408. Acta Physica Hung., 4 (1955), p. 301.

10. Brown, R. H., and Twiss, R. Q., Nature, 177 (1956), p. 27; 178 (1956), p. 1046 ; Proc. Roy. Soc. A., 243 (1957), p. 291.

11 Twiss, R. Q., Little, A. G., and Brown, R. H., Nature, 180 (1957), p. 324.

Rebka. G. A. and Pound. R. V., Nature. 180 (1957) p. 1035.

Brannen, E., Ferguson, H. I. S., and Wehlau, W., Canad. J. Phys., 86 (1958), p. 871.

12 Rice, S. O., in Selected papers on noise in stochastic processes (Dover publications, 1954), pp. 184-227.

13. Born, M., The natural philosophy of cause and chance (Clarendon Press, 1949) p. 79.

Purcell, E. M., Nature, 178 (1956), p. 1449.

14. Jbnossy, L., Nuovo Cimento, 6 (1957), p. 111 also Nuovo Cimento, 12 (1959), p. 369.

16. Forrester, A. T., Gudmundsen, R. A., and Johnson, P. O., Phys. Rev., 96 (1955) p. 1691.

17. Dodd, J. N., Fox, W. N., Series, G. W., and Taylor, M. J., Proc. Phys. Soc. Lond., 74 (1959), p. 789.

18. Heisenberg, W., The physical principles of the quantum theory (University of Chicago Press, 1930). p. 88.

19. Farago, P. S., and M arx, G ., Acta Physica Hung., 4 (1954), p. 23; Phys. Rev., 99 (1955), p. 1063.

20. Smith, L. P., Phys. Rev., 69 (1946) p. 195.

21 Fogarassy, B., unpublished communication to Dr. P. S. Farago.

22. Slepyan, L. B., Radio Engineering, 12 (1957), p. 1.

23. Dicke. R. H.. Rev. Sci. Inst.. 17 (1946). p. 268.

24. Fürth, R., Physica, 17 (1951), p. 259.

25. Sillitto, R. M., Introduction to non-relativistic quantum mechanics (Edinburgh University Press, 1960) chapter 12. 